\title{
The Why of Abandonment: Effects of Team Diversity and Leadership Type on the Disbandment and Stagnation of Online Medical Teams
}

\author{
Jiaying Li \\ Huazhong University of Science \\ and Technology \\ ljyhust@ hust.edu.cn
}

\author{
Hong $\mathrm{Wu}$ \\ Huazhong University of Science \\ and Technology \\ hongwu@hust.edu.cn
}

\author{
Zhaohua Deng* \\ Huazhong University of Science \\ and Technology \\ zh-deng@ hust.edu.cn
}

\begin{abstract}
Medical teams (MTs) online could provide more comprehensive and rapid services to patients through the collaboration among physicians. Numerous doctors have participated, but parts of MTs disband or stagnate after a period, so this pressing issue is in need of relief through exploring the reasons. Effects of team diversity, leadership types and their interaction on the team disbandment and stagnation were studied. This study comprehensively examined a sample of 1,071 MTs online, the total MTs on January 10, 2018, and we crawled the data from a leading OHC in China. Logistic regression was utilized. Results revealed team state would be influenced by team diversity and its interaction with leadership type, so the combination pairwise of the leadership and team diversity could reduce the abandonment possibility. Implications in theory and practice about the dealing with the abandonment crisis in online health community, and limitations are discussed.
\end{abstract}

\section{Key words:}

Medical teams online, Team diversity, Leadership types, Team dissolution, Team stagnation.

\section{Abbreviation:}

Online health communities: OHCs; Medical teams: MTs; Team dissolution: TD;

Team stagnation: TS.

\section{Introduction}

Online medical teams (MTs), a novel form of healthcare service, have emerged in online health communities (OHCs) in 2017. With the increasing popularity, as of early 2019, more than 3000 MTs employing over 10,000 doctor members have been developed in China. To some extent, online MTs can alleviate the current imbalance between medical supply and demand in China. On one hand, doctors can improve the service responsiveness and optimize the resource allocation through communicating and cooperating with others in MTs to alleviate supply issues such as shortage and unreasonable allocation of human resources in healthcare services. On the other hand, population aging and rising living standard have triggered the soaring demands for healthcare service in terms of quantity and quality [1], and MTs in OHCs can meet these high requirements by increasing both of them.

MTs online have been considered theoretically promising because of their advantages, but prompt solutions of serious abandonment issues are needed in practice. Considering the term "abandonment" as being expansively used in various settings, our research attempts to solve this through defining it in this specific MT context, including team dissolution (TD) and team stagnation (TS) that may ultimately lead to TD. According to our data obtained from OHCs, approximately $20 \%$ of MTs have disbanded, more than $20 \%$ of MTs have stagnated, and the number is continuously increasing. There exists several negative effects of TD and TS on the teambased services in OHCs. From a patient's perspective, TD and TS can affect the continuity of medical services. For service providers and the platform, they worsen the relationships between participating doctors and OHCs, subsequently affecting the supply of healthcare services online. In addition, once the state of MTs is stagnant, it is dangerous for doctor members to go slow in teams, and even in OHCs, which is not conducive for the provision and delivery of healthcare services in OHCs.

Virtual teams are hard to run efficiently because their operation and management are complex [2]. Likewise, MTs online have the above experience. Most doctors are inexperienced in the establishment of online MTs, so some teams may face the risk of 
disbandment and stagnation. Once members feel the lack of improvement and have few constraints on stagnating or leaving, they may collectively choose to stagnate or leave [3]. In particular, when members prefer other previous forms of online healthcare services such as written/telephone consultation to MTs, they would insist and even deny the value of team-based services. Therefore, TD and TS depend on a number of factors related to leadership and members.

The lack of research on MT disbandment and stagnation is noteworthy given the recent calls for examining its factors to adopt effective adjustments. Existing studies on virtual teams mostly focus on team performance in other fields [4, 5], but studies concerning TS and TD in the healthcare sphere are relatively scarce and usually disregarded, mainly because most virtual teams are temporary and their stagnation or disbandment is based on task completion [6] without the need for further studies. Whereas, MTs in OHCs are long-term and their TS and TD should be empirically investigated to better understand the teams and achieve their sustainable development.

Studies have focused on the effects of team leadership, team diversity and other aspects on virtual team performance [7-9]. First, some scholars summarized 24 studies on team diversity in different areas, concluding that diversity can significantly affect team output/performance in most cases [10], and lead to conflict and negative effects on teambased services [7-9], so it can further influence TS and TD. Second, the critical roles of leadership in a team have been confirmed [9, 11], and the key influence of the leadership is without exception in the context of MTs. Leaders in MTs should not only consider the status and department match and coordination degrees of members at the beginning, but also adjust a team to improve its adaptability while facing challenges such as stagnation, disbandment and members' withdrawal. Therefore, TD and TS are in need of quantified empirical analysis to understand its actual impact factors from aspects of team diversity and leadership types. Specific research questions are presented as follows:

(1) How does the diversity of online MTs affect team state?

(2) How does leadership types of online MTs affect team state?

This study mainly investigates the influences of team diversity and leadership type on the TD and TS of online MTs. It is organized as follows. We introduce the theoretical background in the second part and the model hypotheses in the third part. Then, we describe the methods and results in the fourth and fifth parts, respectively. Conclusions and implications of this study are discussed in the sixth part.

\section{Theoretical Background}

\subsection{Medical teams and leadership types in OHCs}

Group work is an essential part of modern health care [12]. MTs, composed of a founder and other members, such as nurses, medical technicians, or doctors, provide the "several-to-one" healthcare consultation for a patient in an OHC through communication equipment, thereby realizing both doctor-doctor and doctor-patient communication. MTs are characterized by member dispersion and team virtuality, which are also two principal characteristics of virtual teams [13]. Studies on virtual teams have covered different fields, such as open source software development teams, top management teams, and research and development teams in international corporations $[4,5]$. Only a few studies have explored online MTs and proved their ability to meet the complex needs of patients $[14,15]$, and confirmed the important role of a leader's capital in team output from the transactive memory perspective [16].

Leadership plays a crucial role in adjusting a team to adapt well, especially when it faces obstacles and challenges such as member withdrawal, TS or TD [17-19]. Varying types of leadership function differently [20, 21], so it is indispensable for MTs online to be divided into three coordination modes: integrated, assistant, and independent. In the assistant mode, the leader is considered dominant over members; in the two other modes, the leader and members are regarded equally [16]. However, this classification disregards the existence of the weaktype leadership, so we divide leadership into three types: strong, equal and weak (Figure 2) by comparing clinical titles of team leader and members.

\subsection{Team diversity and related influence}

Scholars have classified team diversity and studied its effect on team performance [10, 22, 23]. Informational diversity referring to task and social category diversity focusing on social demographic indicators should be a common classification [24]. However, given the particularity of online MTs and medical services, we use the classification proposed by Harrison and Klein, and categorize diversity into three: separation, variety and disparity [25]. Specifically, separation diversity focuses on the 


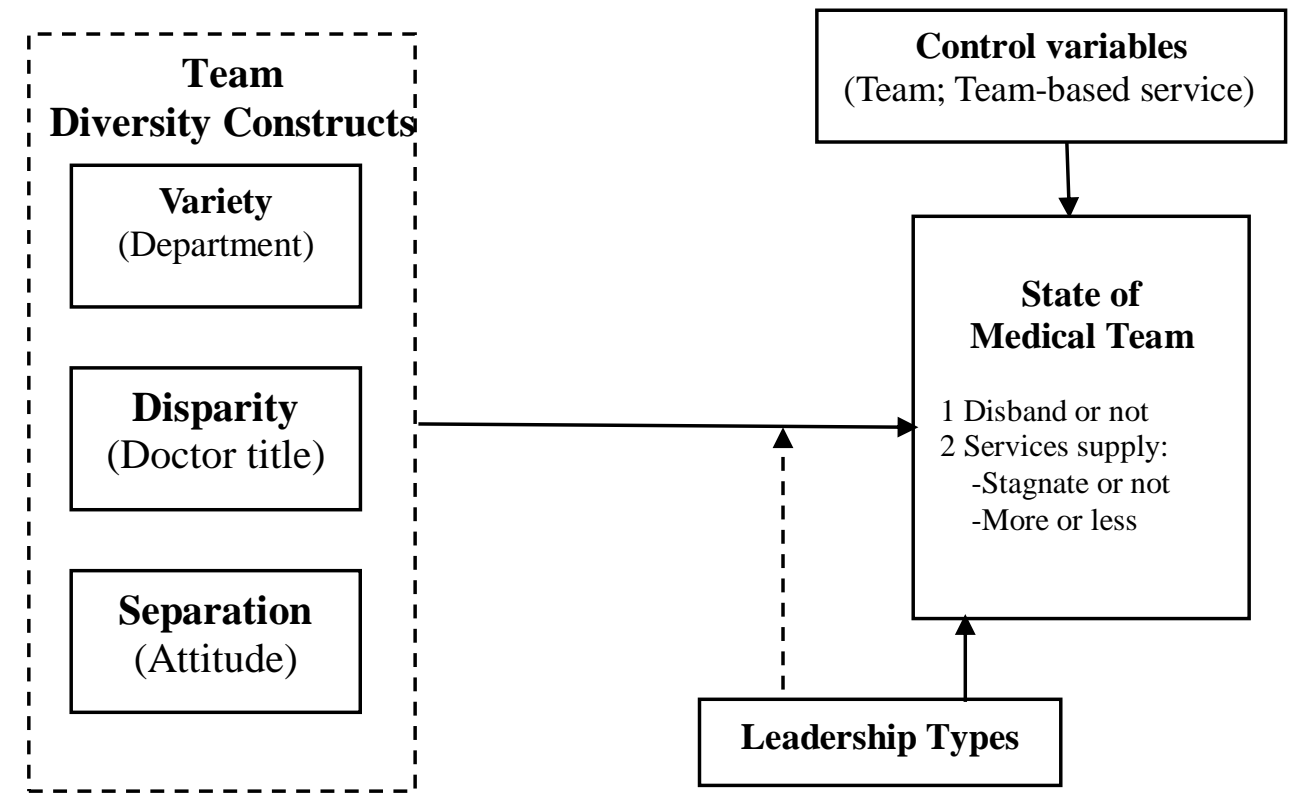

Figure I. Conceptual model

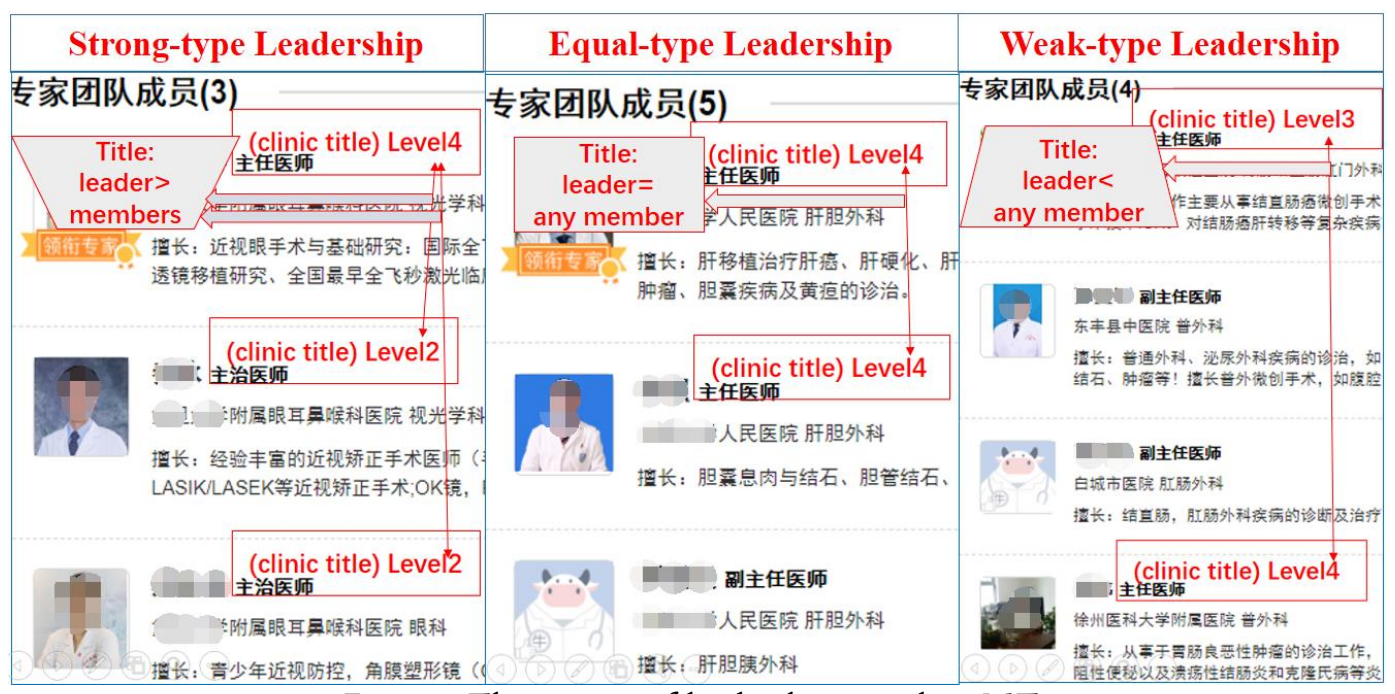

Figure 2 Three types of leadership in online MTs

Note: Team leader is the first person in each MT, and others below are members. 
inconsistency or opposition of members in subjective aspects such as attitude, which can be reflected by the response speed of doctors, as the more positive a doctor in $\mathrm{OHCs}$ is, the faster his/her response to a patient will be. Variety diversity highlights members' differences in professional skills/knowledge and information, which corresponds to distinctive departments of diseases in the medical field. Disparity diversity emphasizes the difference in the vertical continuum of status or power among members. In the $\mathrm{OHC}$ context, patients tend to trust doctors with a high status [26], and the main indicator of doctors' status capital is clinical title [27], so disparity diversity depends on the difference in members' clinical titles. Nevertheless, effects of diversity on team output/performance are inconclusive $[10,25]$, so its influence on the abandonment of MTs should be further studied.

Based on the Social Influence Theory, team diversity refers to the informational influence, while leadership types are related to normative influence. Prior research has proved that peer influence could affect the behavior of doctors in medical teams [28], and this theory has been widely used in online communities and teams [29], so it could apply to this MT context. The social influence of leadership type and team diversity inevitably affect the team state, so we could explain the influences of two factors in our study based on this theory [29].

\subsection{Team disbandment and team stagnation}

Several fragile virtual teams are prone to stagnate or disband, and the reasons remain to be explored. MTs in OHCs have existed in China for almost 2 years, and some teams have disbanded with the increasing number of teams, since members may collectively choose to leave when they feel improvement is unlikely and have few constraints on stagnating or leaving [3]. Scholars have investigated the effect of organizational differences on the technology abandonment from a negative perspective [30], so we also focus on the abandonment of teambased service from the negative perspective. Concerning this topic, organizational characteristics and leadership have been explored [31, 32]. Based on these points, we examine factors of TS and TD in OHCs from perspectives of team diversity and leadership.

\section{Research Model and Hypotheses}

This study focuses on the two perspectives of team diversity and leadership types to explore different team states on the basis of extensive literature and related reviews on virtual teams $[6,17$, 33]. Team diversity often leads to social loafing among members [34]. Team leadership plays a key role in dealing with crises such as disbandment [17, 18]. In addition, according to the social influence theory and related analysis above, the informational influence of team diversity and the normative influence of leadership types both affect the team state. Thus, we choose these two aspects to investigate the state of MTs. The conceptual model is shown in Figure 1. Although team status include TS and TD, we only hypothesis from the TD perspective for briefness in avoid of repetitive statements, so our study mainly focus on the state of TD and just regard TS as a supplement.

\subsection{Team diversity and TD}

As a whole, given the negative effects of three diversity types on team-based services [25], diverse teams are more likely to disband. Members possibly think that their contribution is non-essential in a diverse team, then reducing dispensable efforts; meanwhile, others would also reduce their effort to avoid the "sucker effect" caused by free riders' exploitation [35, 36]. Eventually, this free-riding behavior would lead to the loss of team-based services and a vicious cycle among members in MTs.

Separation diversity, referring to the subjective attitude of members in MTs, can decrease the cohesion among team members, subsequently causing decreased performance [37] and further TD. In addition, differences in members' attitudes can cause distrust and conflicts among members, leading to decreased contribution and even withdrawal of some members [25]. This finding is consistent with the conclusion of social loafing in team research [34]. Thus, we develop the following hypothesis:

\section{Hypothesis 1a: Separation diversity is a risk factor} of TD in OHCs.

In view of the high professionalism in the medical field, variety diversity of members' departments affects the perceived professionalism and disbandment of a team. People tend to choose professional healthcare services [27] and seek the help of MTs online purposefully. Considering medical experience, homogeneous teams that are more likely to result in a professional impression, attracting and retaining more patients than diverse teams do. Only in this virtuous cycle can members be motivated to continue providing services as a team and vice versa, so the following assumption is developed: 
Hypothesis 1b: Variety diversity is a risk factor of TD in OHCs.

Disparity diversity in the MT context affects the team status (i.e., whether or not to disband) through the service speed. Medical services unlike other services refer to the life and sufferings of patients, extremely requiring timeliness. In fact, doctors with high professional titles are busy with services offline, leaving less time for services online, and doctors with low professional titles usually have time online, so differences in members' status result in their complementarity in time. Thus, given the patients' urgent need for disease relief through medical services, the negative effect of social loafing among members is secondary to the positive effect of time complementarity. In addition, previous studies confirmed that disparity could positively affect outcomes by accelerating decision-making [38, 39].Thus, we predict:

Hypothesis 1c: Disparity diversity is a protective factor of TD in OHCs.

\subsection{Leadership type and TD}

Previous studies examined the key role of leadership in team functioning and the different influences of leadership types on team state [21]. Especially, the timely adjustment of leaders is necessary while facing challenges $[17,18]$, so MTs with different leaders have different levels of adaption ability. First, TD usually results from the team's inability to adapt to challenges or remove obstacles, and the weak-type mode is the most likely to result in disbandment because the leader is weakest among three leadership types in clearing obstacles or adapting to challenges. Furthermore, members prefer the strong-type leadership, forming a relatively stable structure to avoid $\mathrm{TD}$, since an implicit social norm suggests that people are willing to obey to authority [40], and a person's behavior is regulated by social norms [41]. Finally, equal-type leadership mode is conducive to form a loose, flexible and democratic atmosphere, where the MT can be easily adjusted to promote the virtuous circle of service and avoid disbanding. Following this logic, we test the following:

Hypothesis 2a (2b): The strong-type (equal-type) leadership is a factor that prevents TD, and the MT with strong-type (equal-type) leadership is not easy to disband compared with that of the weak type.

\subsection{Moderating effect of leadership types}

Many scholars studied the interaction between leadership and team diversity [42, 43]. On the one hand, evidence suggests that team diversity has significant interaction effects with leaders' experience and characteristics [43, 44], indicating that the effect of team diversity varies with leadership types. On the other hand, doctors' high level of clinical title in China suggests the rich clinical experience. Thus, leadership types defined according to the clinical title can reflect doctors' experience to some extent, affecting team diversity. Furthermore, considering the common phenomenon of mutual social compensation among members [34], weaktype leaders tend to compensate for their own weakness by increasing diversity to reduce the TD possibility. However, strong/equal-type leaders are different from a weak-type leadership. Based on the leadership classification and actual situations, strongtype and equal-type leaders in MTs look for equal or less experienced doctors as members, so they subjectively do not need the social compensation from others.

Effects of team diversity on TD changes with leadership types. First, as separation diversity may lead to conflicts or social loafing among members and strong-type leaders usually tend to be busy with medical services offline, these MTs are more likely to lack cohesion and then disband than those with a weak-type leadership. Second, members from diverse departments are indispensable for the weak-type leader, so social compensation exists in these MTs, but high variety diversity are redundant for the other leadership types. Third, given that disparity diversity and strong-type leadership are protective factors against TD, their synergistic effects are essential for the stability of MTs. Thus, we predict the following:

Hypothesis 3: The strong-type leadership positively moderates the relationship between team diversity (i.e., separation diversity (3a), variety diversity (3b), and disparity diversity (3c)) and TD compared with that of the weak-type leadership.

Equal-type leaders function similarly, mainly because they seem relatively dominant or strong compared with weak-type leaders. Equal-type leaders exhibit flexibility to adjust team diversity, so they can positively moderate the relationship between team diversity and team status. Thus, we hypothesize the following:

Hypothesis 4: The equal leadership type positively moderates the relationship between team diversity (i.e., separation diversity (4a), variety diversity (4b), and disparity diversity (4c)) and TD compared with that of the weak-type leadership. 


\section{Methods}

\subsection{Sample and data collection}

We tested our conceptual model in a sample of 1,071 MTs. In this study, data of MTs were crawled from the Good Doctor Online (i.e., www.haodf.com/) website, one of the leading OHCs in China. We studied the change in the status of these teams (whether the team disband/stagnate or not) during a 3-month period from January 10, 2018, to April 10, 2018. At the beginning, a total of 1,071 MTs were available online on January 10, 2018. Thus, the sample was 1,071 MTs and 4,740 doctor members. Table 1 explains the measurement of variables in the empirical models.

\subsection{Measurement}

Dependent Variable. The dependent variable in this study was the status of the MT. The value was 1 when a team disbanded, and the value of undissolved teams was 0. Among the 1,071 MTs, 206 MTs disbanded, accounting for nearly $20 \%$.

Independent Variables. Team diversity and leadership types were two aspects of explanatory variables. Team diversity included separation, variety, and disparity. Separation was mainly reflected by the standard deviation of doctors' attitude toward healthcare service in $\mathrm{OHCs}$, namely, the response speed of individual service. Variety was determined by the number of departments and the status Disparity was demonstrated by the standard deviation of team members' clinical titles. Leadership in MTs can be classified into three categories: strong, equal and weak-type. When the level of leader title was higher than the title levels of other members, the team was defined as strong-type leadership, and the corresponding variable was LTypedummy1. When the level of leader title equaled that of any other member, the MT belonged to an equal or democratic leadership type, and the dummy variable was LTypedummy2. When the level of leader title was lower than that of the other members, the MT leadership was weak (Figure 2). The formula for the two dummy variables of the leadership type was as follows:

LTypedummy $1=\left\{\begin{array}{c}1, \text { team leader title is higher than other members } \\ 0, \text { others }\end{array}\right.$ LTypedummy $2=\left\{\begin{array}{c}1, \text { team leader title is equal to any member } \\ 0, \text { others }\end{array}\right.$

Control Variables. Control variables included indicators of team and team-based service such as team size, response speed, initial service quantity and price. Team size and early stage team activities affect the team status $[16,45]$. Thus, control variables include initial service quantity, price, response speed and team size.

\subsection{Empirical models}

The dependent variable was the status of the MT. The status value of the MT in the dissolution state was 1 , and the status value of the MT that did not disband was 0 . Considering that the dependent variable is a binary variable, we used logistics regression to test the hypothesis.

$$
\begin{aligned}
\text { Ln } \frac{P(\text { TeamStatus }=1 \mid X)}{P(\text { TeamStatus }=0 \mid X)} & =\beta_{0}+\beta_{1} \text { TeamSize }+\beta_{2} \text { ResponseSpeed } \\
& +\beta_{3} \text { InitialServiceQuantity }+\beta_{4} \text { Price } \\
& +\beta_{5} \text { Separation }+\beta_{6} \text { Variety }+\beta_{7} \text { Disparity } \\
& +\beta_{1} \text { LTypedummy } 1+\beta_{9} \text { LTypedummy } 2 \\
& +\beta_{11} \text { LTypedummy } 1 \times \text { Separation } \\
& +\beta_{11} \text { LTypedummy } 2 \times \text { Separation } \\
& +\beta_{12} \text { LTypedummy } 1 \times \text { Variety } \\
& +\beta_{13} \text { LTypedummy } 2 \times \text { Variety } \\
& +\beta_{14} \text { LTypedummy } 1 \times \text { Disparity } \\
& +\beta_{15} \text { LTypedummy } 2 \times \text { Disparity }+\varepsilon
\end{aligned}
$$

\section{Results}

Logistic regression was conducted to test the hypotheses. Maximum likelihood estimation (MLE) was performed to estimate empirical results, and $\mathrm{p}<$ 0.05 was considered statistically significant. Data were analyzed using STATA.

\subsection{Descriptive statistics and correlations}

Descriptive statistics and Pearson correlations for all variables are presented in Table 2. The mean value for status of the MT was 0.19 , which indicated that approximately $20 \%$ of all MTs studied in this paper disbanded during the 3-month period. The VIF value of all variables were less than 10 , so we could ignore multicollinearity [46].

\subsection{Empirical results}

The influences of most variables were significant. To test the hypotheses of the proposed model, we considered five models. First, we only tested the effects of control variables in Model 1. Furthermore, we respectively added Team Diversity and leadership types in Model 2 and Model 3, where we evaluated $\mathrm{H} 1$ and $\mathrm{H} 2$. Finally, we built their 
interaction and tested $\mathrm{H} 3$, and results are shown in Table 3. Hypothesis 1 was partially supported because Hypothesis 1a was not supported. Hypothesis 1b and Hypothesis 1c were supported. Results supported Hypothesis 2, including Hypothesis 2a and Hypothesis 2b. Hypothesis 3 including three hypotheses, and only Hypothesis $3 \mathrm{~b}$ was supported. Results partially supported Hypothesis 4 since only Hypothesis 4b was supported Among the control variables, team size was a risk factor of TD. Response speed and price had no significant influence on TD. InitialServiceQuantity was the protective factor of TD. In addition, team size had no significant influence on TS, other control variables were all protective factors of TS.

Factors have both direct and indirect influences on TD. First, considering the direct influence, Separation Diversity had no significant influence on TD. Variety Diversity and the weak-type leadership were the risk factors of TD. Disparity Diversity and strong- and equal-type leadership were the protective factors against TD. Second, considering the moderating effect, the interaction between Disparity Diversity and leadership types had no significant effect on TD. As separation had no significant influence on TD, we disregarded its interaction with leadership types. Thus, leadership types only significantly affected the relationship between Variety Diversity and TD, and strong/equal-type leadership enhanced the positive effect of Variety Diversity on TD, suggesting that MTs with strong/equal-type leadership should have fewer departments. Following the same logic, MTs online with multiple departments and weak-type leadership were less likely to disband.

\subsection{Post study}

Considering that the intrinsic mechanism of team diversity's influence on team status was consistent, we studied the influence of team diversity on TS. The sample was 865 teams except the disbanded MTs, among which 277 teams are stagnant, and results were shown in table 4. Separation diversity was the protector factor of TS, and disparity diversity works as the risk factor not as expected, primarily because two kinds of diversity were both correlated with time complementarity. Other factors did not function significantly.

We also examined effects of team diversity and leadership types on performance (i.e., the team-based service quantity during 3-month study period) in MTs based on the remaining 588 teams without stagnation from the opposite perspective, and the results were shown in Table 5. The effects of leadership type and status disparity were robust. However, separation had a negative effect and variety did not have a remarkable effect, since it was differently that diversity and leadership may functions at different stages. And while providing services, members with various separation might be faced with more conflict in the team due to their distinctive attitudes. Meanwhile, now that the MT had been chosen, the variety did not matter any more during the service delivery.

\subsection{Robustness tests}

We selected the method of eliminating the extreme values by using a part of the total samples to test the robustness of results. We aimed to study the MTs online that had used this service form, so teams that did not provide team-based service were excluded from samples, and we ran models by disregarding teams whose initial service quantity was zero. We also used the logistic regression to test whether the result was robust. Among these MTs, 78 teams had zero initial service quantity, and the remaining 993 teams had ever provided team-based services. Results were robust according to variable coefficients, odds ratios and significance levels in Table 6.

\section{Discussion}

\subsection{Result analysis}

The results reveal the micro-foundations of the MT state by highlighting team diversity, leadership type and their pairwise combinations. In particular, the potential mechanisms such as the time complementarity in MTs are also highlighted. Furthermore, the normative influence and informational influence function differently in MTs with different types of leadership [47]. Finally, there exits different impacts of the same factor on TD and TS, demonstrating that leadership and team diversity functions differently in different contexts. Consequently, it is necessary to distinguish various kinds of MTs and their state.

\subsection{Implications}

This study contributes to theories as follows. First, this investigation is unique and comprehensive because it empirically studies the abandonment issue of actual MTs, especially the different degree of abandonment such as TD and TS. Second, both the informational influence of team diversity and the normative influence of leadership type are 
emphasized in our study based on the social influence theory, so it offers insights into the potential mechanisms underlying the observed MT state, and enriches the theories in OHCs and the application scope of this theory. Third, it offers some different perspectives of leadership and team diversity classification in online MTs, which is conducive to further research on MTs in OHCs.

In practice, the value of team design has been highlighted [31, 48], and this study could provide related suggestions for team founders/leaders and platform. First, pairwise combinations of leadership types and variety diversity could protect MTs from disbandment. Second, regarding the TS, members are remarkably affected by attitude separation and status disparity, so MTs should avoid the attitude separation and encourage the status disparity among members to ensure the active services in MTs. Third, it is indispensable to differentiate different types of leadership according to their attributes, especially since weak-type leadership is distinctive from other leadership types.

\subsection{Limitation}

This study has several limitations. First, we used a cross-sectional design to investigate the relationship between team structure and TS/TD, further studies could conduct an in-depth study with scientific panel data to confirm the causal relationship. Second, we studied the team status from the perspective of members' diversity that focused on the bottom-up effect [49], and the top-bottom effect to explore the influence of MTs on members should be studied. Accordingly, MTs should be further studied from several directions below. In order to ensure the sustainability of MT development, longitudinal studies focusing on team state, atmosphere and performance are needed. After all, doctors participate in MTs with the purpose of realizing their own benefit or interests, so top-bottom effects such as the effects of MTs on their members' performance or the individual attitude need to be explored.

\section{References:}

[1] Deng, Z., S. Liu, and O. Hinz, "The health information seeking and usage behavior intention of Chinese consumers through mobile phones", Information Technology \& People, 28 (2): pp. 405-423.

[2] García Guzmán, J., et al., "How to get mature global virtual teams: a framework to improve team process management in distributed software teams", Software Quality Journal, December 01, 18 (4): pp. 409-435.
[3] Bartunek, J.M., Z. Huang, and I.J. Walsh, "The development of a process model of collective turnover", Hum Relat, 61 (1): pp. 5-38.

[4] Faraj, S., S. Kudaravalli, and M. Wasko, "Leading collaboration in online communities", MIS Q, 39 (2): pp. 393-412.

[5] Hoegl, M. and H.G. Gemuenden, "Teamwork Quality and the Success of Innovative Projects: A Theoretical Concept and Empirical Evidence", Organization Science, 12 (4): pp. 435-449.

[6] Powell, A., G. Piccoli, and B. Ives, "Virtual teams: a review of current literature and directions for future research", SIGMIS Database, 35 (1): pp. 6-36.

[7] Cohen, S.G. and D.E. Bailey, "What Makes Teams Work: Group Effectiveness Research from the Shop Floor to the Executive Suite", J Manage, 23 (3): pp. 239-290.

[8] Ilgen, D.R., et al., "Teams in Organizations: From Input-Process-Output Models to IMOI Models", Annual Review of Psychology, 56 (1): pp. 517-543.

[9] Johnson, S.L., H. Safadi, and S. Faraj, "The Emergence of Online Community Leadership", Inf Syst Res, 26 (1): pp. 165-187.

[10] Webber, S.S. and L.M. Donahue, "Impact of highly and less job-related diversity on work group cohesion and performance: a meta-analysis", J Manage, 27 (2): pp. 141162.

[11] Ye, H.J., Y. Feng, and B.C.F. Choi, "Understanding knowledge contribution in online knowledge communities: A model of community support and forum leader support", Electronic Commerce Research and Applications, 2015/01/01/, 14 (1): pp. 34-45.

[12] Gennari, J.H., et al., "Asynchronous communication among clinical researchers: A study for systems design", Int. J. Med. Inform., 2005/10/01/, 74 (10): pp. 797-807.

[13] Ortiz de Guinea, A., J. Webster, and D.S. Staples, “A meta-analysis of the consequences of virtualness on team functioning", Inf Manage, 2012/10/01/, 49 (6): pp. 301-308. [14] Verhoef, J., et al., "Pilot study of the development of a theory-based instrument to evaluate the communication process during multidisciplinary team conferences in rheumatology”, Int. J. Med. Inform., 2005/10/01/, 74 (10): pp. 783-790.

[15] Tang, T., et al., "Using an electronic tool to improve teamwork and interprofessional communication to meet the needs of complex hospitalized patients: A mixed methods study”, Int. J. Med. Inform., 2019/07/01/, 127 pp. 35-42.

[16] $\mathrm{Wu}, \mathrm{H}$. and $\mathrm{Z}$. Deng, "Knowledge collaboration among physicians in online health communities: A transactive memory perspective", Int $\mathrm{J}$ Inf Manage, 2019/12/01/, 49 pp. 13-33.

[17] Gilson, L.L., et al., "Virtual Teams Research:10 Years, 10 Themes, and 10 Opportunities", J Manage, 41 (5): pp. 1313-1337.

[18] Baard, S.K., T.A. Rench, and S.W.J. Kozlowski, "Performance Adaptation:A Theoretical Integration and Review", J Manage, 40 (1): pp. 48-99.

[19] Ali, N.a., et al., "Knowledge management systems success in healthcare: Leadership matters", Int. J. Med. Inform., 2017/01/01/, 97 pp. 331-340. 
[20] Ruggieri, S. and C.S. Abbate, "Leadership Style, SelfSacrifice, and Team Identification", Soc Behav Pers, //, 41 (7): pp. 1171-1178.

[21] Goleman, D., "Leadership That Gets Results", Harv. Bus. Rev., 2000, 78 (2): pp. 78-90.

[22] Pelled, L.H., K.M. Eisenhardt, and K.R. Xin, "Exploring the Black Box: An Analysis of Work Group Diversity, Conflict and Performance", Adm Sci Q, 44 (1): pp. 1-28.

[23] Dulebohn, J.H. and J.E. Hoch, "Virtual teams in organizations", Human Resource Management Review, 2017/12/01/, 27 (4): pp. 569-574.

[24] Jehn, K.A., G.B. Northcraft, and M.A. Neale, "Why Differences Make a Difference: A Field Study of Diversity, Conflict and Performance in Workgroups", Adm Sci Q, 44 (4): pp. 741-763.

[25] Harrison, D.A. and K.J. Klein, "What's the difference? diversity constructs as separation, variety, or disparity in organizations", Acad Manage Rev, 32 (4): pp. 1199-1228.

[26] $\mathrm{Wu}, \mathrm{H}$. and N. Lu, "Online written consultation, telephone consultation and offline appointment: An examination of the channel effect in online health communities", Int. J. Med. Inform., 2017/11/01/, 107 pp. 107-119.

[27] Guo, S., et al., "How Doctors Gain Social and Economic Returns in Online Health-Care Communities: A Professional Capital Perspective", J Manag Inf Syst, 2017/04/03, 34 (2): pp. 487-519.

[28] Wu, H. and N. Lu, "How your colleagues' reputation impact your patients' odds of posting experiences: Evidence from an online health community", Electronic Commerce Research and Applications, 2016/03/01/, 16 pp. 7-17.

[29] Hu, X., X. Chen, and R.M. Davison, "Social Support, Source Credibility, Social Influence, and Impulsive Purchase Behavior in Social Commerce", International Journal of Electronic Commerce, 2019/07/03, 23 (3): pp. 297-327.

[30] Greenwood, B.N., et al., "The When and Why of Abandonment: The Role of Organizational Differences In Medical Technology Life Cycles", Manage Sci, 63 (9): pp. 2948-2966.

[31] Suttmoeller, M., "The role of leadership and other factors in the organizational death of domestic far-right extremist organizations(Order No. 3643699).”, ProQuest Dissertations \& Theses Global A\&I: The Humanities and Social Sciences Collection; Social Science Premium Collection. (1609719256). , pp.

[32] Hansson, M., "Organizational closedown and the process of deconstruction and creativity", Culture and Organization, 2017/05/27, 23 (3): pp. 238-256.

[33] Martins, L.L., L.L. Gilson, and M.T. Maynard, "Virtual Teams: What Do We Know and Where Do We Go From Here?”, J Manage, 2004/11/01/, 30 (6): pp. 805-835. [34] Williams, K. and S. Karau, "Social Loafing and Social Compensation: The Effects of Expectations of Co-Worker Performance", J. Pers. Soc. Psychol., 10//, 61 (4): pp. 570581.

[35] Kerr, N.L. and S.E. Bruun, "Dispensability of member effort and group motivation losses: Free-rider effects", J. Pers. Soc. Psychol., 44 (1): pp. 78-94.
[36] Nijstad, B.A. (2009) Group performance. New York : Psychology Press.

[37] Daniel, S., R. Agarwal, and K.J. Stewart, "The Effects of Diversity in Global, Distributed Collectives: A Study of Open Source Project Success", Inf Syst Res, 24 (2): pp. 312-333.

[38] Park, B., J.R. Overbeck, and J. Correll (2005) Internal Status Sorting in Groups: The Problem of too many Stars, in: Status and Groups. 169-199.

[39] Groysberg, B., J.T. Polzer, and H.A. Elfenbein, "Too Many Cooks Spoil the Broth: How High-Status Individuals Decrease Group Effectiveness", Organization Science, 22 (3): pp. 722-737.

[40] Milgram, S., "Some Conditions of Obedience and Disobedience to Authority", Hum Relat, 18 (1): pp. 57-76.

[41] Elster, J., "Social Norms and Economic Theory", J. Econ. Perspect., 3 (4): pp. 99-117.

[42] Groves, K.S. and A.E. Feyerherm, "Leader Cultural Intelligence in Context:Testing the Moderating Effects of Team Cultural Diversity on Leader and Team Performance", Group Organ Manag, 36 (5): pp. 535-566.

[43] Choudhury, P. and M.R. Haas, "Scope versus speed: Team diversity, leader experience, and patenting outcomes for firms", Strategic Management Journal, 39 (4): pp. 9771002.

[44] Buyl, T., et al., "Top Management Team Functional Diversity and Firm Performance: The Moderating Role of CEO Characteristics", Journal of Management Studies, 48 (1): pp. 151-177.

[45] Der Foo, M., P. Kam Wong, and A. Ong, "Do others think you have a viable business idea? Team diversity and judges' evaluation of ideas in a business plan competition", J Bus Ventur, 2005/05/01/, 20 (3): pp. 385-402.

[46] Alin, A., "Multicollinearity", Wiley Interdiscip Rev Comput Stat, 2 (3): pp. 370-374.

[47] Deutsch, M. and H.B. Gerard, "A study of normative and informational social influences upon individual judgment", The Journal of Abnormal and Social Psychology, 51 (3): pp. 629-636.

[48] Burns, L.R. and D.R. Wholey, "Adoption and Abandonment of Matrix Management Programs: Effects of Organizational Characteristics and Interorganizational Networks", Acad Manage J, 36 (1): pp. 106-138.

[49] Kozlowski, S.W.J. and K.J. Klein (2000) A multilevel approach to theory and research in organizations: Contextual, temporal, and emergent processes, in: Multilevel theory, research, and methods in organizations: Foundations, extensions, and new directions., Jossey-Bass: San Francisco, CA, US. 3-90. 
Table 1. Descriptive statistics.

\begin{tabular}{|c|c|c|}
\hline Variables & Explanation & Type \\
\hline \multicolumn{3}{|l|}{ Dependent variables } \\
\hline Team_status & 1 if a medical team disbanded; 0 otherwise. & Dummy \\
\hline \multicolumn{3}{|l|}{ Independent variables } \\
\hline \multicolumn{3}{|l|}{ Diversity constructs } \\
\hline Separation & The standard deviation of all members' response speed & Interval \\
\hline Variety & The total number of the doctor departments in a MT & Interval \\
\hline \multirow[t]{2}{*}{ Disparity } & The standard deviation of doctors' clinic titles in a MT. Clinic titles include four levels: Resident Doctor, Attending Doctor, Associate Chief Doctor, & Interval \\
\hline & Chief Doctor from low to high level, correspondingly the value of CTitle is from 1 to 4. & \\
\hline \multicolumn{3}{|l|}{ Leadership type } \\
\hline LTypedummyl & 1 if the title of team leader is highest among all members (strong-type leadership); 0 otherwise. & Dummy \\
\hline LTypedummy2 & 1 if when the title of team leader is equal to the title of any member in medical team (equal-type leadership); 0 otherwise. & Dummy \\
\hline \multicolumn{3}{|l|}{ Control variables } \\
\hline \multicolumn{3}{|l|}{ (Team Service) } \\
\hline TeamSize & The number of team members & Interval \\
\hline ResponseSpeed & Response speed of the team-based written consultation & Interval \\
\hline InitialServiceQuantity & The initial service quantity of a MT & Interval \\
\hline Price & Price offered by the MT for the team-based written consultation & Interval \\
\hline
\end{tabular}


Table 2. Descriptive statistics (including Pearson correlations) ( $\mathrm{n}=1071)$.

\begin{tabular}{|c|c|c|c|c|c|c|c|c|c|c|c|c|c|c|}
\hline Variables & Min. & Max. & Mean & S.D. & VIF & (1) & (2) & (3) & (4) & (5) & (6) & (7) & (8) & (9) \\
\hline (1) Status of the MT & 0 & 1 & 0.19 & 0.39 & - & & & & & & & & & \\
\hline (2) Separation & 0 & 0.5 & 0.31 & 0.17 & 1.082 & 0.038 & & & & & & & & \\
\hline (3) Variety & 1 & 21 & 1.62 & 1.31 & 1.438 & $0.393^{* *}$ & $0.106^{* *}$ & & & & & & & \\
\hline (4) Disparity & 0 & 1.9 & 0.31 & 0.19 & 1.398 & $-0.166^{* *}$ & $0.090^{* *}$ & $-0.138^{* *}$ & & & & & & \\
\hline (5) LTypedummy1 & 0 & 1 & 0.59 & 0.49 & 3.468 & $-0.319^{* *}$ & -0.055 & $-0.321^{* *}$ & $0.432^{* *}$ & & & & & \\
\hline (6) LTypedummy2 & 0 & 1 & 0.32 & 0.47 & 3.294 & $0.153^{* *}$ & 0.060 & $0.231^{* *}$ & $-0.420^{* *}$ & $-0.827^{* *}$ & & & & \\
\hline (7) TeamSize & 2 & 24 & 4.43 & 2.80 & 1.582 & $0.205^{* *}$ & $0.248^{* *}$ & $0.492^{* *}$ & $0.118^{* *}$ & $-0.269^{* * *}$ & $0.246^{* *}$ & & & \\
\hline (8) ResponseSpeed & 0 & 1 & 0.41 & 0.44 & 1.097 & -0.053 & $0.083^{* *}$ & 0.039 & 0.021 & -0.042 & $0.060^{*}$ & $0.120^{* *}$ & & \\
\hline (9) InitialServiceQuantity & 0 & 636 & 12.21 & 32.18 & 1.097 & $-0.064^{*}$ & 0.034 & 0.019 & $0.101^{* *}$ & 0.018 & -0.024 & $0.106^{* *}$ & $0.272^{* *}$ & \\
\hline (10) Price & 9 & 800 & 82.23 & 88.32 & 1.020 & 0.041 & $0.106^{* *}$ & $0.078^{*}$ & 0.019 & -0.018 & 0.006 & $0.101^{* *}$ & 0.037 & 0.0 \\
\hline
\end{tabular}

Notes: **. Correlation is significant at the 0.01 level (2-tailed); *. Correlation is significant at a 0.05 level (two tailed). 
Table 3. Results of hierarchical logistic regression $(\mathrm{N}=1071)$.

\begin{tabular}{|c|c|c|c|c|c|c|c|c|}
\hline \multirow[t]{2}{*}{ Variables } & \multicolumn{2}{|c|}{ Model 1} & \multicolumn{2}{|l|}{ Model 2} & \multicolumn{2}{|c|}{ Model 3} & \multicolumn{2}{|l|}{ Model 4} \\
\hline & coefficient & $\operatorname{Exp}(B)$ & coefficient & $\operatorname{Exp}(B)$ & coefficient & $\operatorname{Exp}(B)$ & coefficient & $\operatorname{Exp}(B)$ \\
\hline Intercept & $-2.083(0.170)^{* * * *}$ & 0.125 & $-2.185(0.264)^{* * * *}$ & 0.113 & $-0.610(0.346)^{*}$ & 0.544 & $1.256(0.732)^{*}$ & 3.512 \\
\hline TeamSize & $0.182(0.027)^{* * * *}$ & 1.199 & $0.087(0.038)^{* *}$ & 1.091 & $0.063(0.040)$ & 1.065 & $0.062(0.041)$ & 1.064 \\
\hline ResponseSpeed & $-0.248(0.199)$ & 0.781 & $-0.306(0.217)$ & 0.736 & $-0.307(0.222)$ & 0.736 & $-0.331(0.225)$ & .718 \\
\hline InitialServiceQuantity & $-0.016(0.006)^{* * *}$ & 0.984 & $-0.013(0.006)^{* *}$ & 0.987 & $-0.012(0.006)^{* *}$ & 0.988 & $-0.013(0.006)^{* *}$ & .987 \\
\hline Price & $0.000(0.001)$ & 1.000 & $0.000(0.001)$ & 1.000 & $0.000(0.001)$ & 1.000 & $0.000(0.001)$ & 1.000 \\
\hline Separation & & & $-0.035(0.540)$ & 0.966 & $-0.009(0.561)$ & 0.991 & $-2.781(1.396)^{* *}$ & .062 \\
\hline Variety & & & $0.694(0.083)^{* * *}$ & 2.001 & $0.603(0.086)^{* * *}$ & 1.828 & $0.251(0.157)^{*}$ & 1.285 \\
\hline Disparity & & & $-2.217(0.561)^{* * * *}$ & 0.109 & $-1.456(0.646)^{* *}$ & 0.233 & $-2.470(1.670)^{*}$ & .085 \\
\hline LTypedummy1 & & & & & $-2.044(0.277)^{* * * *}$ & 0.130 & $-4.636(0.897)^{* * *}$ & .010 \\
\hline LTypedummy2 & & & & & $-1.432(0.276)^{* * *}$ & 0.239 & $-3.554(0.814)^{* * *}$ & .029 \\
\hline LTypedummy $1 *$ Separation & & & & & & & $2.877(1.661)^{*}$ & 17.762 \\
\hline LTypedummy $2 *$ Separation & & & & & & & $3.715(1.646)^{* *}$ & 41.057 \\
\hline LTypedummy $1 *$ Variety & & & & & & & $0.861(0.239)^{* * *}$ & 2.365 \\
\hline LTypedummy $2 *$ Variety & & & & & & & $0.304(0.183)^{*}$ & 1.355 \\
\hline LTypedummy1* Disparity & & & & & & & $0.833(1.959)$ & 2.300 \\
\hline LTypedummy $2 *$ Disparity & & & & & & & $1.470(1.877)$ & 4.350 \\
\hline -2Log likelihood & 991.707 & & 863.130 & & 809.496 & & 788.158 & \\
\hline Chi-square & 57.023 & & 185.601 & & 239.234 & & 260.573 & \\
\hline Correct Percentage & 0.814 & & 0.824 & & 0.836 & & 0.840 & \\
\hline
\end{tabular}

Notes: $N=1071$. Standard errors are in parentheses. MLE. $* \mathrm{p}<0.1 . * * \mathrm{p}<0.05 . * * * \mathrm{p}<0.01$ 
Table 4. Results of hierarchical logistic regression $(\mathrm{N}=\mathbf{8 6 5})$.

\begin{tabular}{lcccc}
\hline \multicolumn{1}{c}{ Variables } & \multicolumn{2}{c}{ Model 1 } & Model 2 \\
\cline { 2 - 5 } & coefficient & $\mathbf{E x p}(\mathbf{B})$ & coefficient & $\mathbf{E x p ( B )}$ \\
\hline Intercept & $0.812(0.202)^{* * *}$ & 2.252 & $0.872(0.283)^{* * * *}$ & 2.392 \\
TeamSize & $-0.07(0.040)$ & 0.993 & $0.007(0.045)$ & 1.007 \\
ResponseSpeed & $-1.140(0.231)^{* * *}$ & 0.320 & $-1.106(0.23)^{* * *}$ & 0.331 \\
InitialServiceQuantity & $-0.208(0.029)^{* * *}$ & 0.813 & $-0.210(0.029)^{* * *}$ & 0.810 \\
Price & $-0.003(0.001)$ & 0.997 & $-0.003(0.001)^{* *}$ & 0.997 \\
Separation & & & $-0.927(0.516)^{*}$ & 0.396 \\
Variety & & & $-0.091(0.120)$ & 0.913 \\
Disparity & & & $0.811(0.479)^{*}$ & 2.250 \\
-2Log likelihood & 791.386 & & 784.625 & 300.161 \\
Chi-square & 293.400 & & 0.777 & \\
Correct Percentage & 0.769 & & & \\
\hline
\end{tabular}

Notes: $N=1071$. Standard errors are in parentheses. MLE. $* \mathrm{p}<0.1 . * * \mathrm{p}<0.05 . * * * \mathrm{p}<0.01$. 
Table 5. Results of hierarchical linear regression $(\mathrm{N}=588)$.

\begin{tabular}{lcccccc}
\hline \multicolumn{1}{c}{ Variables } & Model 1 & \multicolumn{3}{c}{ Model 2 } & \multicolumn{3}{c}{ Model 3 } \\
\cline { 2 - 7 } & coefficient & S.E. & coefficient & S.E. & coefficient & S.E. \\
\hline Intercept & $4.564^{* * *}$ & 1.401 & $3.229^{*}$ & 1.902 & -0.514 & 3.266 \\
TeamSize & $-0.414^{*}$ & 0.226 & $-0.421^{*}$ & 0.253 & $-0.587^{* *}$ & 0.261 \\
ResponseSpeed & 1.921 & 1.380 & 1.994 & 1.370 & 1.633 & 1.371 \\
InitialServiceQuantity & $0.310^{* * *}$ & 0.014 & $0.305^{* * *}$ & 0.014 & $0.307^{* * *}$ & 0.014 \\
Price & $0.022^{* * *}$ & 0.007 & $0.022^{* * *}$ & 0.007 & $0.022^{* * *}$ & 0.007 \\
Separation & & & $-6.210^{*}$ & 3.728 & $-6.543^{*}$ & 3.726 \\
Variety & & & 0.179 & 0.679 & 0.190 & 0.691 \\
Disparity & & & $9.483^{* * *}$ & 2.966 & $12.911^{* * *}$ & 3.354 \\
LTypedummy1 & & & & & 2.708 & 2.738 \\
LTypedummy2 & & & & & $5.939^{* *}$ & 2.809 \\
$\mathbf{R}^{2}$ & 0.471 & & 0.482 & & 0.488 & \\
Adjusted $\mathbf{R}^{\mathbf{2}}$ & 0.467 & & 0.476 & & 0.480 & \\
$\mathbf{F}$ & 129.765 & & 77.122 & & 61.286 & \\
$\Delta \mathbf{R}^{2}$ & 0.471 & & 0.011 & & 0.006 & \\
$\Delta \mathbf{F}$ & 129.765 & & 4.138 & & 3.516 & \\
\hline
\end{tabular}

Notes: $N=1071$. S.E. represents standard errors. OLS. ${ }^{*} \mathrm{p}<0.1 . * * \mathrm{p}<0.05 . * * * \mathrm{p}<0.01$. 
Table 6. Robustness test results $(N=993)$.

\begin{tabular}{|c|c|c|c|c|c|c|c|c|}
\hline \multirow[t]{2}{*}{ Variables } & \multicolumn{2}{|c|}{ Model 1} & \multicolumn{2}{|l|}{ Model 2} & \multicolumn{2}{|c|}{ Model 3} & \multicolumn{2}{|l|}{ Model 4} \\
\hline & coefficient & $\operatorname{Exp}(B)$ & coefficient & $\operatorname{Exp}(B)$ & coefficient & $\operatorname{Exp}(\mathbf{B})$ & coefficient & $\operatorname{Exp}(\mathbf{B})$ \\
\hline Intercept & $-1.837(0.174)^{* * *}$ & 0.159 & $-1.809(0.271)^{* * *}$ & 0.164 & $-0.305(0.354)$ & 0.737 & $1.463(0.746)^{*}$ & 4.320 \\
\hline TeamSize & $0.174(0.027)^{* * * *}$ & 1.190 & $0.080(0.038)^{*}$ & 1.083 & $0.059(0.040)^{*}$ & 1.061 & $0.059(0.041)$ & 1.061 \\
\hline ResponseSpeed & $-0.423(0.200)$ & 0.655 & $-0.488(0.218)^{*}$ & 0.614 & $-0.480(0.224)^{*}$ & 0.619 & $-0.494(0.226)^{*}$ & .610 \\
\hline InitialServiceQuantity & $-0.018(0.006)^{* * * *}$ & 0.982 & $-0.015(0.007)^{* *}$ & 0.985 & $-0.014(0.007)^{* *}$ & 0.986 & $-0.015(0.006)^{*}$ & .986 \\
\hline Price & $0.000(0.001)$ & 1.000 & $0.000(0.001)$ & 1.000 & $0.000(0.001)$ & 1.000 & $0.000(0.001)$ & 1.000 \\
\hline Separation & & & $-0.534(0.560)$ & 0.586 & $-0.476(0.584)$ & 0.622 & $-2.691(1.412)^{*}$ & .068 \\
\hline Variety & & & $0.699(0.084)^{* * *}$ & 2.012 & $0.610(0.088)^{* * * *}$ & 1.841 & $0.255(0.159)^{*}$ & 1.291 \\
\hline Disparity & & & $-2.182(0.566)^{* * *}$ & 0.113 & $-1.475(0.651)^{*}$ & 0.229 & $-2.764(1.696)^{*}$ & .063 \\
\hline LTypedummy1 & & & & & $-1.986(0.281)^{* * *}$ & 0.137 & $-4.500(0.906)^{* * * *}$ & .011 \\
\hline LTypedummy2 & & & & & $-1.389(0.281)^{* * * *}$ & 0.249 & $-3.361(0.828)^{* * *}$ & .035 \\
\hline LTypedummy $1 *$ Separation & & & & & & & $2.259(1.695)$ & 9.578 \\
\hline LTypedummy $2 *$ Separation & & & & & & & $2.294(1.682)^{*}$ & 19.096 \\
\hline LTypedummy $1 *$ Variety & & & & & & & $0.871(0.244)^{* * * *}$ & 2.388 \\
\hline LTypedummy $2 *$ Variety & & & & & & & $0.299(0.186)^{*}$ & 1.349 \\
\hline LTypedummy1* Disparity & & & & & & & $1.182(1.972)$ & 3.260 \\
\hline LTypedummy $2 *$ Disparity & & & & & & & $1.829(1.910)$ & 6.226 \\
\hline -2Log likelihood & 954.948 & & 828.051 & & 778.263 & & 759.367 & \\
\hline Chi-square & 59.027 & & 185.924 & & 235.712 & & 254.608 & \\
\hline Correct Percentage & 0.819 & & 0.819 & & 0.834 & & 0.835 & \\
\hline
\end{tabular}

Notes: $N=993$. Standard errors are in parentheses. MLE. $* \mathrm{p}<0.1 . * * \mathrm{p}<0.05$. $* * * \mathrm{p}<0.01$ 